\title{
Traversées migratoires: Des étoiles (2013) de Dyana Gaye
}

\section{Monleón-Domínguez, Ana}

Universitat de València, ana.monleon@uv.es

\begin{abstract}
Resumen
Recuperado por la literatura y el cine, el fenómeno de la inmigración clandestina y de las migraciones generales constituye hoy día un corpus específico. Una nueva tipología de relatos y de personajes que presenta en su organización características comunes han ampliado las literaturas de expresión francesa. Volveremos a encontrar esta intención de dar visibilidad a las experiencias de los migrantes en algunas películas que representan esta realidad para la gran pantalla. Dyana Gaye parte de estas premisas y elabora una nueva versión de estas referencias transformando el espacio de las migraciones, por lo general cerrado, en una dinámica abierta en la que los viajes y los movimientos inscriben a los personajes en el contexto intercultural de los desplazamientos migratorios actuales.
\end{abstract}

Palabras clave : Inmigración cladestina ; movimientos migratorios ; interculturalidad ; Des étoiles ; Dyana Gaye.

\section{Résumé}

Récupéré par la littérature et par le cinéma, le phénomène de l'immigration clandestine et des migrations générales constitue de nos jours un corpus spécifique. Une nouvelle typologie de récits et de personnages, présentant dans leur organisation de base des caractéristiques communes et fixes qui ont élargi le champ de la représentation des littératures d'expression française. Nous retrouverons la même intention de donner une visibilité aux situations que traversent les migrants dans certains films qui reconstruisent ces réalités pour l'écran. Dyana Gaye, tout en partant de ces références, réélabore ces données en transformant l'espace des migrations, habituellement clos, en une dynamique ouverte où les voyages et les déplacements des personnages les situe dans le contexte interculturel des mouvements migratoires actuels.

Mots-clés : Immigration clandestine ; mouvements migratoires ; interculturalité ; Des étoiles ; Dyana Gaye.

\begin{abstract}
Gathered by literature and cinema, the phenomenon of clandestine immigration and common migrations constitutes nowadays a specific corpus. A new type of stories and characters which show in their basic organisation common and fixed characteristics that have increased the representation field of French expression literatures. We will find the same intention of giving visibility to situations the migrants suffer in many films that reconstruct those realities for the big screen. Starting from those references, Dyana Gaye, reformulates these data transforming migrations spaces, usually closed, to an open dynamic where the travels and shifts of the characters locates them in the intercultural context of the current migrational movements.
\end{abstract}

Keywords : clandestine migration ; migrational movements ; interculturality ; Des étoiles ; Dyana Gaye. 
Dans cette approche au premier long métrage de Dyana Gaye, Des étoiles, nous voulons mettre en valeur le déplacement que la réalisatrice opère par rapport aux textes ou encore aux films, qui à partir des années 2000, prennent en charge la représentation des migrations clandestines. Dyana Gaye renvoie, d'ailleurs, à cette thématique en situant, cependant, son film bien plus sur l'axe du mouvement, que sur les repères textuels que génèrent les récits sur l’immigration. Nous aurons l'occasion de revenir sur cette réflexion de l'auteure dans les lignes qui suivent.

\section{La brûlure de l'eau}

\subsection{Récits sur l'immigration clandestine}

En ce qui concerne les textes portant sur l'immigration, nous mentionnerons, entre autres, des récits tels que le Néant Bleu (2000) de Did El Hamri ou encore Il était parti dans la nuit (2004) de Youssef Amghar. Pour ce qui est des films citons : Harragas (2009) de Merzak Allouache, Clandestins (1997) de Denis Chouinard et Nicolas Wadimoff, Welcome (2009) de Philippe Lioret, Nulle part, terre promise (2008) d'Emmanuel Finkiel. Et finalement nous pouvons ajouter également à ce corpus constitué de nombreuses références, l'album du rappeur d'origine congolaise Abdel Al Malik : Gibraltar (2006).

Tous les ouvrages que nous venons de mentionner, avec des styles différents, partagent un dénominateur commun qui pourrait-être exprimé au moyen de l'image de «la brûlure de l'eau » que nous posons en reprenant l'expression algérienne «h'rag », mot de l'arabe dialectal désignant ces jeunes qui brûlent leurs papiers pour éviter de se faire reconnaître par la police espagnole et par conséquent se faire reconduire dans leurs pays d'origine. La brûlure, dans l'acception que nous lui donnons à partir des éléments constitutifs des récits sur l'immigration, est l'acte irréversible qui plonge volontairement l'immigré clandestin dans la dépersonnalisation, l'anonymat absolu et qui voue son être existant à la seule faveur d'un destin qui le confronte à un élément majeur : la mer, la barrière ou encore frontière naturelle qui sépare les deux rives entre l'espace de la misère et celui d'un prétendu confort. Obstacle contre lequel ces clandestins ne sont que faiblement préparés, navigant sur des embarcations frêles ou encore ne sachant pas nager.

Au-delà de tout ce que l'imagination peut se représenter, par ailleurs ces écrivains construisent leurs narrations à partir de témoignages, il n’y a pas à notre connaissance de récits élaborés à partir d'une expérience personnelle de la traversée, les récits amènent les différentes phases de ces voyages au bout du désespoir et de la mort. La décision, qui est en quelque sorte l'acceptation d'une probabilité de mort isole le futur voyageur, car il le faut bien. De la même manière que celui-ci brûlera tout document qui signalerait son identité, sa provenance aux autorités, le clandestin doit également «brûler » tout rapport d'émotions affectives à son entourage qui pourrait nuire à son projet. Phase d'une « quarantaine » affective que le migrant clandestin s’inflige. Le voyageur clandestin largue les amarres, coupe les attaches de son humanité bien avant d’accomplir le voyage. Il sera déjà, avant la traversée, ce noyé potentiel échoué sur une plage de galets ou de sable.

Le voyage par mer est un élément essentiel de ces récits. On en retrouvera de très amples descriptions dans ce type de récits. Ces passages tentent de traduire le dénuement extrême de ces personnes qui défient tous les dangers au risque de leur vie. L’image de la mer «bouche » insatiable qui engloutie, qui dévore ces êtres est récurrente ainsi que l'obstination de ce désir à surmonter les rouleaux de vagues afin d'atteindre la terre ferme. Derrière, il n'y a que le soleil calcinant qui cloue les êtres à la misère et le plus souvent dans un contexte d'oppression. Ces deux éléments de la nature possédant la même force d'anéantissement cèle le rite sacrificiel du clandestin : mourir pour mourir, il choisit d'accomplir ce rite de vie et mort :

Soudain le passeur jeta un coup d'œil furtif sur les mains de sa victime, toujours crispées au bord la barque ....il crut que sa main droite éclatait, puis à sa gauche le bâton venait de s'abattre sur ses doigts. K jeta un cri si terrifiant qu'on dit le hurlement d'un fauve. Son corps s'était effondré dans la mer. Il se débattait de toutes ses forces contre les lames géantes. Mais l'eau pénétrait sa bouche, cravatait sa gorge ; il toussait à s'en déchirer les poumons. Puis il serrait les dents, contractant les mâchoires comme pour soulever un énorme poids. Dans cette lutte tellement inégale, l'effort de l'homme paraissait misérable, infini et vain. K était perdu au milieu du désert liquide ; il avait beau regarder dans toutes les directions, il ne parvenait pas à distinguer le nord du sud, l'est de l'ouest; il avait perdu tous ses repères. Une peur sans nom le submergeait, en l'inondant d'écume. Il ne savait ni où aller, ni vers quel point se diriger, et il ne 
pouvait demeurer immobile. Il suffoquait, nageant contre les vagues dévorantes. Le vent qu'il avalait à grandes goulées l'étouffait presque et, dans la visibilité quasi nulle, ses yeux fouillaient l’ombre sans déceler le moindre signe (El Hamri Rachid, 2005 : 63-65).

\subsection{Quelques films sur l'immigration clandestine}

La deuxième partie du film Harraga de Merzak Allouache se déroule entre ciel et mer. Le bleu du ciel et de la mer se fondant pour créer un contexte irréalisant, sans limites et sans repères. Littéralement les personnages se diluent entre ces deux espaces dans lesquels ils errent à la dérive. On assiste alors à la perte des repères temporels, de leur repère identitaire, et finalement au spectacle d'une dépersonnalisation. Ce qui préfigure aussi les situations qu'ils traverseront à leur arrivée en Europe, pour ceux qui arriveront à surmonter cette barrière liquide.

Dans Welcome de Philippe Lioret, la rencontre d'un français de Calais, dans la cinquantaine, séparé de sa femme qui, elle, a refait sa vie d'un point de vue sentimental sur le point de signer son divorce et qui fait la connaissance d'un jeune adolescent iraquien qui a quitté son pays en guerre pour rejoindre la jeune fille qu'il aime et qui est déjà installée avec sa famille à Londres.

Deux amours, l'un frustré et l'autre dans la splendeur et la fougue de la jeunesse, sont le point de rencontre qui unit ces deux personnages. Et deux mentions à l'eau, le bassin de la piscine dans laquelle travaille le Calaisien en apprenant à nager aux enfants, exercices aquatiques, également, pour des groupes de femmes âgées et autres activités semblables. Le bassin de la piscine, eau domestiquée, à l'image des commodités que les européens peuvent se procurer et l'autre masse liquide immense, houleuse de l'océan atlantique du canal de la Manche.

Ce sont les deux références pour l'adolescent : l'apprentissage de la natation, le crawl, le désir de traverser le canal pour l'amour qu'il porte à la jeune fille. En contrepoint, la fascination du Calaisien pour ce projet inouï, lui qui n'est pas arrivé à retenir sa femme. Le jeune iraquien apprendra finalement à nager, et fera pratiquement deux fois la traversée. Il n’accomplira pas finalement

\section{Des étoiles: une géopoétique de l'immigration}

Dyana Gaye est une jeune réalisatrice qui a commencé à produire en 2000. Diplômée en études cinématographiques de l’Université de Paris 8. En 1999, elle est lauréate de la bourse d'étude Louis Lumière - Villa Médicis Hors les Murs pour son scénario Une femme pour Souleymane ${ }^{1}$, qu'elle réalisera l'année suivante, film qui obtient de nombreux prix dans des festivals internationaux. Parallèlement, elle travaille pendant plusieurs années comme programmatrice pour l’ACID (Agence pour le Cinéma Indépendant pour sa Diffusion).

En 2004, elle réalise J'ai deux amours pour le projet « Paris la métisse », série de 15 courts-métrages, et elle est finaliste du programme Rolex de mentorat artistique. En 2006, son court-métrage Deweneti 2 connaît une large diffusion nationale et internationale. Il reçoit le Prix du Jury au Festival International du Film de Clermont-Ferrand en 2007 et fait partie des cinq films nommés aux Césars 2008 du meilleur court-métrage. Elle produit ensuite Un transport en commun (2009), comédie musicale présentée au Festival de Films de Locarno en compétition "Cinéastes du présent », et sélectionnée entre autres aux festivals de Sundance et de Toronto. Il fait partie des cinq films nommés aux César 2011 du meilleur court-métrage. C’est en 2013, qu'elle réalise Des étoiles, premier long-métrage tourné entre Dakar, Turin et New-York qui sera présenté en première mondiale au Festival de Toronto dans la section Contemporary World Cinema.

\footnotetext{
${ }^{1}$ Dans ce court métrage, la réalisatrice présente la situation de Souleymane Diallo, un jeune Sénégalais habitant à Paris depuis trois ans et qui tente de surmonter sa solitude. Pour ne pas décevoir les siens, il préfère leur mentir, inventant dans ses lettres une vie d’employé de banque et une fiancée nommée Jeanne. Tout le monde s'en réjouit à Dakar. En réalité, Souleymane est serveur dans une brasserie, et sa seule confidente est Machami, une fillette astucieuse. Adama Diallo, 18 ans, sœur cadette de Machami, trouve suspect le peu de nouvelles et d'enthousiasme de Souleymane. Il avait promis de la faire venir en France. Le film montre les angoisses et la détresse du personnage dans le piège d'une double vie.

${ }^{2}$ Le court métrage focalise Ousmane, un petit garçon talibé, qui mendie dans les rue de Dakar et qui sans savoir écrire veut envoyer une lettre au Père Noël pour lui demander d'exaucer les vœux des personnages de son entourage. Choc entre deux cultures, deux mondes riche et pauvre dont la valeur est inversement proportionnelle dans l'optique de l'enfant qui ne possédant rien ne demande que le bonheur des autres à ce Père Noël qui se transforme dans son imagination en une sorte de dieu qui peut pourvoir aux nécessités des Dakarois.
} 
Le film reçoit le Grand prix du Jury et le prix du public au Festival Premiers Plans Angers 2014. Finalement en 2014, elle réalise Un conte de la goutte d'or, court-métrage musical pour les Talents Adami Cannes.

Les critiques que reçoivent ses travaux, et les prix qu'elle obtient depuis le début de sa carrière, font d'elle une réalisatrice prometteuse.

En ce qui concerne ce premier long métrage, Des étoiles, Dyana Gaye le définit dans le Making off du CD comme un film plus sur le mouvement, le transit des personnages, de visages anonymes qui caractérisent le monde actuel. Il y a comme nous l'avons dit, un rapport à ce contexte de l'immigration clandestine, cependant le film met l'accent sur le mouvement, déplacements des personnages et les points de rencontre qu'ils effectuent. Elle utilise, pour définir son film, l'image d'un train en marche qui déversent un flot de gens, les croisant et qui font surgir dans cette intersection des profils d'existence éloignés de la dureté et du pessimisme des textes et films des années 2000. En ce sens le film de Dyana Gaye libère le personnage de la bipolarité spatiale des récits sur l’immigration dans laquelle il se trouvait pris.

En effet les espaces et leur valeur ont été généralement utilisés selon des axes de confrontations qui peuvent être résumés sous les signes de la richesse Vs. pauvreté, domination vs. liberté, déchéances vs. dignité, etc. D’autre part encore, c'est à des espaces fonctionnalisés et typifiés auxquels nous avons affaire généralement. Lieux de départs et de destinations qui inscrivent une toponymie de l'immigration, ces espaces ne parlent pas ou peu du personnage, ne l'exprime pas dans son mouvement intérieur, dans les transformations qu'il subit.

Signalons également que pour la réalisatrice la construction de ce film renvoie à des expériences personnelles et familiales qui rattachent cette création en quelque sorte à son vécu :

\begin{abstract}
Je suis partie de Souki et Malick, deux des personnages d'Un transport en commun, mon précédent film. Souki allait à l'enterrement de son père quand Malick s'apprêtait à émigrer en Italie. Je souhaitais prolonger cette idée du voyage, des trajectoires que l'on trace dans une existence. Je suis vraiment partie de la jeunesse en mouvement et des parcours de l'exil; j’avais aussi une envie précise de travailler sur un personnage féminin, Malick s'est donc transformé en Sophie. Tout cela entre en résonance avec mon histoire. Mon père est sénégalais, il est arrivé en France par la musique au début des années 1970. Ma mère est une métisse franco-italo-malienne-sénégalaise, qui a grandi entre la France, l'Italie et le Sénégal. Mon grand-père paternel était boursier de l'Etat sénégalais et a été envoyé en France à 16 ans, au Lycée Louis-le-Grand, juste après la génération Senghor, Césaire. Ses propres parents avaient fui le Mali pour s'installer au Sénégal au début du siècle dernier. Dans le Paris d'après-guerre, mon grand-père fréquentait beaucoup les cercles littéraires de Saint-Germain des-Prés, (Genet, Prévert) et les clubs de Jazz... C’est à cette époque qu'il a rencontré ma grand-mère qui arrivait d'Italie. Il a été le premier noir dans une petite localité du Nord de l’Italie près de Bergame, ce qui évidemment ne passait pas inaperçu. Je suis née à Paris mais l’italien est ma langue maternelle après le français. J'ai toujours eu un rapport d' « étrangeté » avec ce pays mais aussi une très grande familiarité. Tourner en Italie était une évidence (Entretien avec Dyana Gaye).
\end{abstract}

Nous retrouvons ainsi dans l'ascendance de la réalisatrice des mouvements de migrations. C'est donc un contexte qui lui est familier par ce qu'elle a pu apprendre autour d'elle et qui s'inscrit dans la réalité historique, sociale et culturelle en mentionnant les cercles littéraires de St. Germain des Prés et plaçant les noms de Senghor et Césaire ainsi que le jazz. Nous n'avons pas pour le présent travail l'occasion de nous pencher sur l'utilisation de la musique, des chansons qui couvrent le réseau musical du film, mais notons toutefois que celui-ci répond au souci de représenter l'interculturalité au moyen d'un assemblage particulièrement vivant du playlist du film.

Dyana Gaye établit dans son film un dialogue entre trois villes où les cultures ne se confrontent pas mais s'expriment et se parlent à travers les personnages principaux. Soucieuse également de rendre crédible le jeu des acteurs, elle les choisit soigneusement en rapport étroit avec l'espace dans lequel ils auront à interpréter et représenter leur personnage :

Chaque acteur a vécu d'une certaine manière le déplacement de son personnage. Marème Demba Ly (Sophie) n’était jamais allée en Europe, Ralph Amoussou (Thierno) découvrait le Sénégal et Souleymane Seye N’Diaye (Abdoulaye) New York. Chacun appréhendait ces lieux à la fois dans son personnage et dans son être, ce qui constituait une 
alchimie particulièrement intéressante ; la rencontre d'une altérité. L'incarnation qu'ils avaient à accomplir pour le film rencontrait leur intimité (Entretien avec Dyana Gaye).

\section{Dakar-Turin-New York: Abdoulaye-Sophie-Thierno}

Le film met en place ainsi un contraste sur trois villes qui servent de toile de fond au récit des déplacements et de l'évolution des personnages : entre Dakar, Turin et New York, les destins de Sophie (Marème Demba Ly), Abdoulaye (Souleymane Seye N’Diaye) et Thierno (Ralph Amoussou) se croisent et s'entremêlent, comme les rails du chemin de fer. Des premières désillusions aux rencontres décisives, leur voyage les mènera à faire le choix de la liberté. Les villes représentées dans le film acquièrent également une valeur nouvelle en ce sens qu'il s'établit des rapports d'interactions différentes de celles-ci aux personnages principaux. Les villes de Turin et de New York convergent sur le même sens qui fait d’elles des espaces de la modernité. Nous constatons ici un déplacement important car les personnages sont bien plus motivés par une quête identitaire que par une nécessité urgente de recherche de moyens de survie. Ils vivent, traversent, s’approprient ces villes ou encore errent dans ces deux villes selon le projet qui oriente leur vie.

Abdoulaye est le personnage qui montre un déplacement erratique qui le fait voyager de Dakar à Turin passant ensuite en France, il arrive finalement à New York. C’est le seul personnage du film qui pourrait être rattaché au destin du type d'immigré clandestin dont parlions au début de ces pages. Fermé au monde, l'expression de son visage est marquée par une tristesse empreinte de dureté. À son dépaysement intérieur, à l'inconsistance de ses valeurs morales correspond un voyage qui est l'équivalent d'une fuite et d'une errance. Les raisons pour lesquelles il quitte Dakar sont diffuses ; le spectateur est en mesure de les rattacher au cadre de l'immigration, cependant très vite il aura remplacé son épouse qu'il a quittée à son départ de Dakar pour une autre femme à Turin qu'il abandonnera à son tour, pour aller à New York dans le but de trouver un travail mieux rémunéré. Le choix de New York répond à cette double motivation que nous avons pu constater dans les réflexions de la réalisatrice à propos de son film; à nouveau une inscription personnelle en consonance avec le paysage des migrations actuelles car New York est une ville fréquentée par la réalisatrice. Ville lumineuse pour elle et source d’inspiration, elle représente une référence culturelle faite également d'intersections diverses. De plus, New York « demeure une sorte de point de fixation et d'ancrage pour la figure du migrant, un lieu d'invention avec sa part fantasmatique. Cette idée perdure, ce qui confère à la ville un côté toujours poétique et séduisant. » (Entretien avec Dyana Gaye. http://www.commeaucinema.com/notes-de-prod/des-etoiles,287533)

Cependant Abdoulaye tourne le dos à New York, espace de croisées interculturelles. Il n'est pas en mesure de s’ouvrir à la vie. La plupart du temps il fixe un regard neutre et opaque vers nulle part. Les plans dans le métro qui le mène à son travail le montre de profil alors que par la vitre l'on peut voir le port et ses docks qui, dans l'aménagement industriel, ont domestiqué l'océan. Il se dirigera juste avant son départ pour la Californie sur une promenade maritime. Vu de dos le personnage échappe au spectateur livré à la contemplation du mouvement des vagues de l'océan. Abdoulaye échappe et fuit vers la Californie, comme le lui avait indiqué un homeless avec lequel il partage un renfoncement de porte pour passer la nuit dans la rue, dans le but d'accomplir son rêve de richesse matérielle, mais surtout pour échapper au devoir de reconnaissance et de confiance qui l'a fait trahir la générosité d’un vieux dakarois qui l'aide à New York. C’est un personnage à la dérive, sans attaches qui a perdu progressivement ses traces identitaires sans parvenir à substituer ce manque par d'autres signes susceptibles de le construire en tant qu'être humain.

Sophie et Vadim (Adreï Zayats) pour leur part évoluent intégralement dans le récit du film dans l'espace européen et à Turin concrètement. Dyana Gaye apporte les raisons de ce choix qui retombe sur cette ville italienne en complétant les informations rapportées dans la citation antérieure :

Dans l'histoire de l'Italie, Turin a une place intéressante puisqu'elle a été une ville d'accueil pour une immigration intérieure liée à l'industrialisation d'après-guerre. Je trouvais cet écho émouvant, fort symboliquement, et en résonance avec New York, où l'on trouve notamment «Little Italy » mais aussi « Little Senegal »... Depuis une quinzaine d'années, c'est aussi à Turin que j'ai pu observer une immigration largement féminine - aussi bien d'Afrique que d'Amérique latine ou d'Europe de l'Est - et un tissu associatif et culturel très dense en relation avec les migrants. Avec l'hiver, l'enclavement lié aux montagnes qui l'entoure et son architecture imposante, c'était particulièrement fort de plonger Sophie dans cette ville dont elle ne connaît ni les codes ni la langue (Entretien avec Dyana Gaye). 
Sophie part de Dakar à Turin pour y retrouver Abdoulaye. Elle traversera les différents espaces du circuit des migrants dans la ville de Turin. Espaces officiels d'accueil où elle rencontre Ada (Maya Saisa), aide sociale pour les nouveaux migrants arrivant dans la ville. C'est à grâce à celle-ci qu'elle parviendra à surmonter ses réticences à abandonner les traditions qui la rivent à un mari infidèle dans l'espoir que ce dernier prendra la prendra en charge. Grâce au soutien qui lui est dispensé elle arrive finalement à trouver un travail, apprend l'italien. Le spectateur peut ainsi contempler l'évolution positive du personnage qui fait d'elle une femme autonome, pouvant disposer de son argent.

L'autre espace de solidarité et d'entre aide entre migrants qu'elle connaîtra est le salon de coiffure de Rose qu'elle visite en cherchant les traces du passage de son mari ne sachant pas qu'elle s'adresse à la maîtresse de son mari. L'hostilité de cette première rencontre se transformera par la suite en une relation de complicité féminine dans laquelle Sophie gagne finalement une place dans une relation d'égalité avec les autres femmes et très particulièrement avec Rose.

Cependant Sophie réussira à se défaire des conflits qui l'accompagnaient à son arrivée à Turin. Le souvenir d'Abdoulaye, son mari, s'estompe et laisse place à nouvel horizon amoureux. L'expression de la joie et du bonheur de vivre sur son visage est déjà bien loin aussi du sentiment de crainte et de méfiance qui la caractérisait au début de son séjour dans la ville italienne. Ainsi le démontre la dernière séquence du film qui nous renvoie le reflet du jeune couple formé par Sophie et Vadim. Ils sont dans un train qui doit les mener à Gênes « pour aller faire un tour à la mer » comme Vadim avait suggéré. La mer dans ce cas appartient au registre de la plénitude amoureuse. Ces plans consacrent le succès de la jeune femme qui rejoint la symbolique du mouvement enfin mis à l'œuvre et qui lui a permis de s'éloigner des entraves qui l'immobilisait. Située de plus dans le wagon d'un train, la séquence dégage une impression de dynamisme et de mouvement grâce aux paysages fuyants qui défilent à travers la fenêtre. À l'opposé du trajet de l'aéroport en autobus vers Turin où l'on perçoit l'expression absente et tendue ne regardant pas les paysages qui défilent à la vitre de l'autobus. Finalement le gros plan de la main noire de Sophie dans celle blanche de Vadim scelle la nouvelle direction dans le destin de ces deux personnages.

Le voyage que réalise Thierno à Dakar accompagnant sa mère pour assister à l'enterrement de son père représente le mouvement inverse que les exemples d'Abdoulaye, de Sophie et Vadim nous ont donné. Venant de New York, Thierno représente la deuxième génération dans sa famille. L’adolescent afro-américain découvre la réalité dakaroise et plonge dans le passé de sa famille maternelle qu'il vient de connaître. Il possède également deux prénoms, Thierno pour ce qui est de son identité sénégalaise et Tyler qui le rattache à l’identité du pays dans le lequel il est né et a grandi, les États Unis. Alors que pour Abdoulaye l'enjeu est couper tous les liens avec son passé et tandis que Sophie, qui éprouve de la nostalgie toutefois pour la ville de Dakar, et Vadim mettent à distance leur famille et se réinventent à Turin, pour Thierno-Tyler il s'agira au contraire de découvrir et de tendre un pont entre son identité afro-américaine et celle de sa famille sénégalaise.

C'est d'ailleurs dans cette intention que sa mère lui fait faire ce premier voyage au Sénégal pour qu'il «puisse découvrir son pays ». Le regard que pose l'adolescent sur Dakar est pleinement ouvert. Il se laisse imprégner par tout ce qui l'environne et prend contact sans transition avec les habitudes de sa famille maternelle dans la scène où Aminata sa mère distribue tous les cadeaux qu'elle a rapportés de New York. Parmi ceux- ci, en clin d'œil à l'actualité politique des États-Unis un T-shirt avec l'impression du visage du président Barack Obama.

Thierno est suivi depuis son arrivée à l'aéroport jusqu'au moment où il prépare sa valise et nous assisterons avec lui à tous les événements importants pour sa famille; l'enterrement du père, dont il ne garde que très peu de souvenirs comme il le dira à sa cousine Dior (Johana Kabou), selon le rite musulman se déroule avec la seule présence des hommes qui accompagne le défunt au cimetière. La cérémonie des condoléances réunit un groupe nombreux de personnes et permet à Thierno de faire la connaissance de Dieynaba Fall, la seconde épouse de son père, et à son grand étonnement il rencontre également Adya et Bakary, ses jeunes demi-frères de onze et neuf ans. Surprise également pour les deux enfants qui savaient que leur père avait séjourné aux États-Unis mais n’y croyaient pas trop pensant que c'était encore une histoire inventée par les adultes.

En tant qu'adolescent Thierno-Tyler permet également d'ouvrir la perspective des jeunes sénégalais. C'est ainsi que l'on assiste à la rencontre culturelle des deux mondes improvisée lorsqu'il effectue une visite dans la classe de sa cousine Dior. Questionné par les garçons et les filles de la classe, il répondra qu’il n’est pas marié, étant trop jeune 
encore, et que de plus n'est pas obligatoire aux États-Unis pour former un couple ; qu'il ne connaît pas personnellement le président Barack Obama car un président ne peut pas se rencontrer facilement dans la rue ; qu'il étudie dans une école d’informatique mais que sa passion c'est la musique. Et finalement, répondant à la professeure, il dira que ce qui l'a le plus impressionné de Dakar ce sont les odeurs. Réponse qui provoque l’hilarité générale des élèves prenant la réponse dans le sens le moins poétique évidemment.

Toutes les différentes expériences que traverse Thierno dans ce premier séjour à Dakar présentent une grande naturalité et sont emboîtées par le ressort de la curiosité et de l'étonnement. En aucun cas ne surgissent des réactions hostiles, des préjugés ou encore des refus. Les personnages évoluent dans une atmosphère fluide et non contraignante.

La dernière séquence, très significative, dans laquelle est situé Thierno, juste avant son départ, correspond à la visite à l'île de Gorée qu'il effectue avec sa cousine Dior, déjà bien intéressée par ce beau cousin américain. Le petit voyage en bateau qui les éloigne du port de Dakar avec la rencontre d'un couple afro-américain de Washington D.C et prise de photo, prend l'apparence d'une excursion pour touristes. Vue de loin, l'île de Gorée entourée par l'étendue miroitante de l'océan ressemble à une jolie carte postale exotique. Cependant à l'arrivé sur l'île la voix d'un guide fixe la perspective historique de la colonisation et la traite des noirs signalant aux visiteurs que ce sont les Portugais qui en 1767 ont édifié les bâtiments et indique ainsi la maison des esclaves. Dior et Thierno s’éloignant du groupe découvre la porte d'entrée donnant sur le petit quai d'où l'on faisait descendre les esclaves des bateaux pour les introduire dans l'enceinte du fort.

Les deux jeunes adolescents, de dos, encadrés par l'embrasure de l'étroite porte et regardant le bleu de l'Océan traduisent, dans l'organisation spatiale de la scène le questionnement postcolonial attaché à l'espace historique de l’immigration et des migrations. L'efficacité narrative de Dyana Gaye déplace le spectateur au gré d'un montage très agile sur différentes perspectives humaines, géographiques et historiques.

\section{Références bibliographiques}

AMGHAR, Youssef (2004). Il était parti dans la nuit. Paris : L’Harmattan

El HAmRI, Rachid (2005). Le néant bleu. Paris : L’Harmattan

Entretien avec Dyana Gaye. <http://www.commeaucinema.com/notes-de-prod/des-etoiles,287533> [Consulté le 15 avril 2016 ].

GAYE, Dyana (2012). Des étoiles (film). Dist. Haut et Court.

Redouane, Najib (2008).Clandestins dans le texte maghrébin de langue française. Paris : L’Harmattan. 